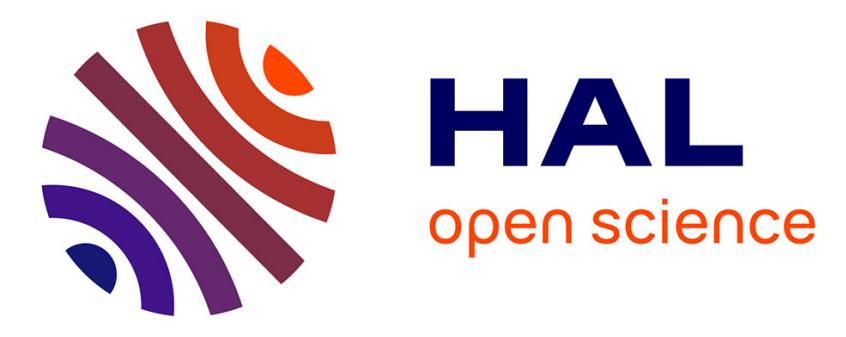

\title{
Three dimensional mobile wireless sensor networks redeployment based on virtual forces
}

Nadia Boufares, Ines Khoufi, Pascale Minet, Leila Saidane, Yosra Ben Saied

\section{To cite this version:}

Nadia Boufares, Ines Khoufi, Pascale Minet, Leila Saidane, Yosra Ben Saied. Three dimensional mobile wireless sensor networks redeployment based on virtual forces. Wireless Communications and Mobile Computing Conference (IWCMC), Aug 2015, Dubrovnik Croatia. pp.563-568 10.1109/IWCMC.2015.7289145 . hal-01250613

\section{HAL Id: hal-01250613 \\ https://hal.inria.fr/hal-01250613}

Submitted on 5 Jan 2016

HAL is a multi-disciplinary open access archive for the deposit and dissemination of scientific research documents, whether they are published or not. The documents may come from teaching and research institutions in France or abroad, or from public or private research centers.
L'archive ouverte pluridisciplinaire HAL, est destinée au dépôt et à la diffusion de documents scientifiques de niveau recherche, publiés ou non, émanant des établissements d'enseignement et de recherche français ou étrangers, des laboratoires publics ou privés. 


\title{
Three Dimensional Mobile Wireless Sensor Networks Redeployment Based On Virtual Forces
}

\author{
Nadia BOUFARES* ${ }^{*}$ Ines KHOUFI ${ }^{\dagger}$, Pascale MINET ${ }^{\dagger}$, Leila SAIDANE* and Yosra BEN SAIED ${ }^{\ddagger}$ \\ *National School for Computer Science, Manouba, Tunisia \\ $\dagger$ Inria, Rocquencourt, 78153 Le Chesnay Cedex, France \\ $\ddagger$ University of Carthage, Tunisia \\ nediya.boufares@gmail.com, ines.khoufi@inria.fr, pascale.minet@inria.fr, \\ leila.saidane@ensi.rnu.tn,yosra_bensaied@yahoo.fr
}

\begin{abstract}
In many applications such as precision agriculture (fruit tree plantation, olive groves) or environmental monitoring, wireless sensors are, very often, randomly scattered in the 3D area of interest. Such applications require full three-dimensional coverage. Undoubtedly, an initial random deployment does not achieve neither full coverage of the $3 D$ area of interest, nor network connectivity. Thus, a redeployment algorithm has to be introduced in order to ensure these two goals. Our contribution is the design of 3D-DVFA, a distributed deployment algorithm based on virtual forces in three dimensional wireless sensor networks where sensor nodes are assumed to be mobile and autonomous.
\end{abstract}

Index Terms-WSN; 3D space; autonomous deployment; coverage; connectivity; virtual forces.

\section{Context And Motivation}

Wireless Sensor Networks (WSNs) are a set of sensor nodes, small devices which have the task of monitoring their environment by detecting the event occurring in the area considered and then reporting the data gathered to the sink. A sensor node may be either mobile and autonomous, or static. Thus, a self deployment is needed to organize autonomous sensor nodes, whereas an assisted deployment (by humans or mobile robots) is needed to place static nodes in their appropriate positions.

In the literature, most studies use a two-dimensional space to model the area considered. However, in the real environment the area to be monitored is not always flat. Also, in some applications where the third dimension, the height, should not be neglected, 2D monitoring is not sufficient. Among the applications which require $3 \mathrm{D}$ monitoring we can cite: home automation, precision agriculture (fruit tree plantation, olive groves), environmental monitoring. The 3D area monitoring differs from $2 \mathrm{D}$ one, then recent studies try to cope with $3 \mathrm{D}$. However, the goal of both $2 \mathrm{D}$ and $3 \mathrm{D}$ monitoring is still to ensure the required coverage (i.e. full or partial coverage of an area, a barrier or a point of interest) and maintain network connectivity such that there is at least one path from each sensor node deployed to the sink.

Our goal is to ensure full 3D area coverage while maintaining network connectivity. Hence, each detected event in the 3D area considered can be reported to the sink. We are interested

978-1-4799-5344-8/15/\$31.00 (c) 2015 IEEE in the 3D deployment of wireless sensor nodes meeting these properties.

Over the last years, researchers focused mainly on theoretical studies, especially on static 3D networks in which the best node placement is predetermined to ensure the desired results. But few studies are based on autonomous and mobile sensor nodes to design a deployment algorithm. In this paper, we assume that sensor nodes are mobile and able to move progressively toward their final positions. Sensor nodes are initially scattered in an arbitrary manner within the area to be controlled which may not fit coverage and connectivity requirements. Then, a redeployment algorithm must be designed to satisfy both area coverage and network connectivity. That is why we propose to design a threedimensional distributed redeployment algorithm whose aim is to redeploy sensor nodes in the 3D space of interest in order to ensure area coverage and network connectivity. This algorithm is based on the virtual forces approach [1].

This paper is organized as follows: In section II, we give an overview of the state of the art. Section III describes in details our three-dimensional distributed virtual forces algorithm, referred as 3D-DVFA. Section IV deals with the performance evaluation of our algorithm. Finally, we conclude in Section VI mentioning future work.

\section{RELATED WORK}

The position of each node in WSN is very critical in the monitoring task. It may have an impact on the data collected. Thus, the deployment task is a fundamental issue in WSNs. Recently, autonomous sensor nodes have emerged in many studies since sensor nodes are able to move thanks to the last technological advances.

Many studies are interested in 2D sensor nodes deployment. To be more realistic, some of them take into account the presence of obstacles and area borders when modeling the deployment algorithms. However, the computation of node positions is based only on the two dimensional area. In the literature, many deployment strategies are proposed. We can cite: virtual forces-based strategy, grid-based strategy and computational based strategy [2]. Generally, the computation 
of the appropriate sensor node positions ensures the required area coverage and maintains network connectivity. However, when the deployment is based on the triangular tesselation and the condition $R_{c} \leq \sqrt{3} R_{s}$ is satisfied, where $R_{c}$ is the communication range and $R_{s}$ is the sensing range, full area coverage ensures network connectivity too [3]. Under this constraint, the deployment algorithm will focus only on the coverage purpose since network connectivity will be a consequence.

The 2D deployment is well studied in the literature. However, few studies focused on the 3D deployment. In [4], the authors highlight the feasibility of extending some existing 2D solutions to $3 \mathrm{D}$ approaches. For a better sensor deployment, a study of different various critical transmitting/sensing ranges to ensure 3D area coverage and guarantee network connectivity is proposed in [5]. In [6], the authors propose a polynomial time solution to satisfy that point of interest is covered by at least $n$ sensors. The value of $n$ should be defined based on the coverage degree required.

Since the number of sensor nodes is very important in the deployment task, authors in [7] and [8] focused on how to minimize the number of sensor nodes needed to cover the $3 \mathrm{D}$ area considered. The authors in [8] propose a node activity scheduling solution to minimize the number of sensor nodes active in the network. Initially, sensor nodes are randomly deployed and only nodes which are placed in the points of interest needed to be covered are switched to active state. The others are switched to sleep state to save energy and prolong network lifetime.

In 2D area coverage, the triangular tesselation was proved to be the optimal strategy in terms of sensor nodes number needed. This property cannot be generalized in 3D area coverage due to the big difference between the two 2D and $3 \mathrm{D}$ deployment problems. However, an interesting study is realized in [9]. The authors demonstrated that the use of Voronoi tessellation to create truncated octahedron cells is the best strategy to achieve full coverage of 3D area using the minimum number of nodes. This deployment requires that the communication range must be at least 1.7889 times the sensing range in order to ensure network connectivity. The study was derived from Kevin conjecture. Other patterns including rhombic dodecahedron, hexagonal prism and cube, are presented too, with their corresponding placement approaches. However, as Kevin and Kepler conjecture, the optimality proof for truncated octahedron tessellation is still not proven.

A further set of studies was achieved in [10] and [11]. Bai and Zhang designed the best patterns to be fit into 3D space and proved their optimality for both area coverage and network connectivity for the first time in [11].

The strategies presented in the previous references are limited to three-dimensional static wireless sensor networks, based on either random or deterministic strategies. Unfortunately, related work focused on deployments of mobile nodes, to our knowledge, were rarely referred. In [12], authors used for the first time the virtual forces strategy to deploy 3D mobile wireless sensor network where each sensor node is able to move in $3 \mathrm{D}$ space according to the virtual forces.

\section{Three Dimensional Distributed Virtual Force ALGORITHM}

In this section, we start by presenting the different assumptions adopted to model a three-dimensional distributed virtual forces algorithm. Then, we detail the main changes brought to the $2 \mathrm{D}$ virtual forces algorithm to deal with $3 \mathrm{D}$ environment and we present the main principles of our 3DDVFA algorithm.

\section{A. Assumptions}

In this paper we adopt the following assumptions in order to ensure full 3D area coverage and network connectivity.

- Each node in the sensor network can determine its own position from global positioning system (GPS).

- The binary sensing model will be adopted as the sensing model, which means the probability of detecting the target is 1 if this one is within the sensor's sensing range, and 0 otherwise.

- The sensing and transmission ranges are deterministic and spherical, generally a common precondition in all $3 \mathrm{D}$ contributions, thus generating sensing sphere and transmission sphere respectively.

\section{B. Applying Distributed Virtual Forces strategies to 3D space}

The improvement made in WSNs enabled the emergence of new networks referred to Mobile WSNs. These mobile WSNs are meeting the requirement of more realistic application requirements such that 3D applications which present our focus in this paper.

Some 2D deployment approaches could be extended to perform in 3D space. Our contribution consists in extending the $2 \mathrm{D}$ virtual forces strategy presented in [1] and [13] to carry out in 3D space. The idea is to take advantage of the virtual forces principles that encourage the spreading of nodes in the whole area while maintaining network connectivity. Traditional virtual forces solutions are inspired from the potential field [1] and disk packing approaches [14].

Generally, sensor nodes are randomly deployed in the 3D area considered. This deployment, may not ensure the required 3D area coverage and some nodes may be disconnected from the sink. Thus, in such a deployment, network connectivity cannot be maintained. By applying the virtual forces strategy, nodes will move according to the virtual forces exerted on them. Progressively, the whole area is covered and network connectivity is still maintained.

To define the virtual forces paradigm, each sensor node is considered as a source of forces on its neighbors. there are two kinds of forces called the attractive and repulsive forces, respectively. These forces are exerted according to the distance between two neighboring sensor nodes. If this distance is higher than the distance threshold $D t h$, then an attractive force is exerted. If it is smaller than $D t h$, then a 
repulsive force is exerted. Otherwise, the exerted force is null. Each node moves according to the resultant force. Figure 1 illustrates an example of virtual forces exerted on sensor $S_{1}$.

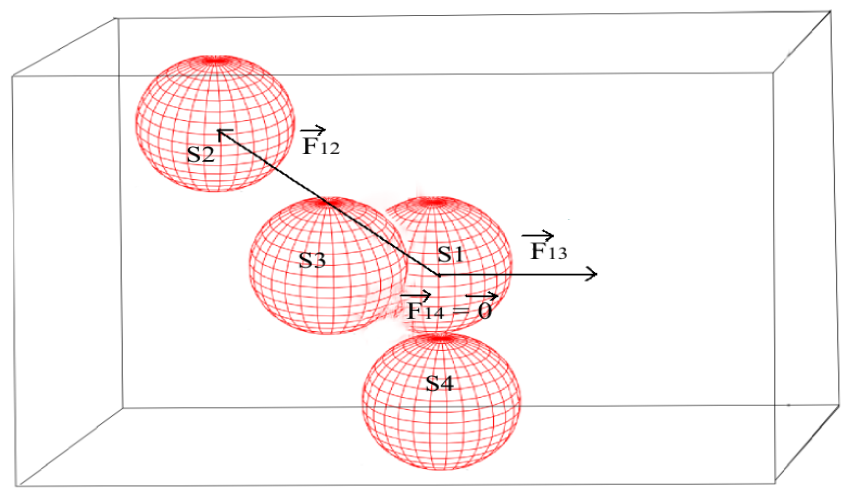

Fig. 1: Example of 3D virtual forces exerted on node S1

Since the role of Dth is very important in the principle of the virtual forces, it should be well tuned. In 2D deployment, Dth is computed according to the optimal deployment based on the triangular tessellation where each node, located at a triangle vertex, has 6 neighbors. However, in 3D space, the optimized deployment is provided by the truncated octahedron tessellation [9]. The truncated octahedron (see Figure 2b) has 14 faces, among them 8 are regular hexagons, whereas 6 are squares. Then, a node has 14 neighbors. If the neighbors are adjacent on a square face, the target distance is equal to $4 R_{s} / \sqrt{5}$. In contrast, if they are adjacent on an hexagonal face, the target distance is $2 R_{s} \sqrt{3} / \sqrt{5}$. Then, when the truncated octahedron is used, two target distances are maintained between neighboring nodes depending on their respective positions.

However, since in the virtual forces strategy, only one target distance is maintained between neighboring nodes, we do not adopt the truncated octahedron tessellation as a deployment pattern. We prefer a regular tesselation requiring a unique $D t h$. The regular dodecahedron(see Figure 2a) is a regular polyhedron composed of 12 equally sized regular pentagons. Since it is regular, a node in the center of the dodecahedron has 12 neighbors at the same distance. In this work, we adopt the regular dodecahedron to determine the target distance in order to apply the virtual forces strategy. See for instance, Figure 3 that shows the regular dodecahedron tesselation where the node in the center of the dodecahedron has 12 neighbors.

Let $a$ be the edge length of a regular dodecahedron. The radius of the circumscribing sphere that intersects the dodecahedron at all vertices, represents the sensing range $R_{s}$ and is equal to:

$$
R_{s}=a \frac{\sqrt{3}}{4}(1+\sqrt{5}) .
$$

The target distance is equal to:

$$
D t h=a \sqrt{\frac{5}{2}+\frac{11}{10} \sqrt{5}}
$$

For instance, in our performance evaluation (see Section IV), the edge length $a$ is equal to $10 \mathrm{~m}$.

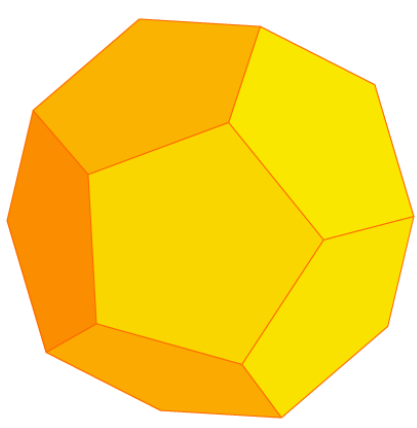

a Regular Dodecahedron.

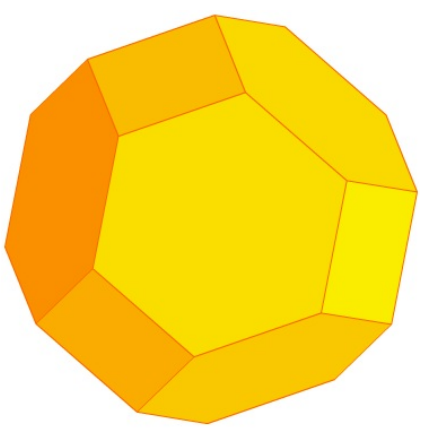

b Truncated octahedron.
Fig. 2: 3D geometric shapes.

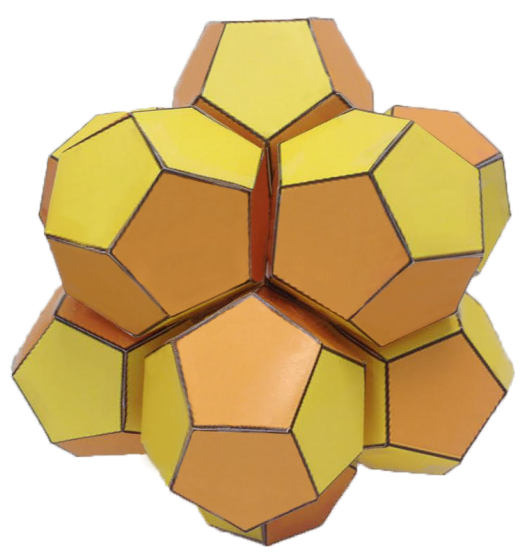

Fig. 3: Regular Dodecahedron tesselation

Based on the principle of the virtual forces meeting the requirements of 3D environment, we design a deployment algorithm called 3D-DVFA for Three Dimensional Distributed Virtual Forces Algorithm. The 3D-DVFA is a distributed algorithm that ensures uniform 3D area coverage and guarantees network connectivity. Moreover, it is adapted to almost all 3D environments since the 3D assumptions and principles defined fit with almost all 3D applications, mentioned previously.

\section{The 3D-DVFA principles}

We now detail how 3D-DVFA works. Each sensor node within the network runs the following algorithm that proceeds by iterations but does not require node synchronization. Let $s_{i}$ denote any sensor node and $\left(x_{i}, y_{i}, z_{i}\right)$ be its coordinates. At each iteration, each node broadcasts a Hello message. In the Hello message, each node sends its position and the node it hears in order to perform the neighborhood discovery. Then, each sensor node is able to determine its 1-hop neighbors and 2-hop neighbors, and compute its new position according to the forces exerted on itself by its 1-hop and 2-hop neighbors.

Let $d_{i j}$ denote the euclidean distance between the sensor nodes $s_{i}$ and $s_{j} . d_{i j}$ is given by $\sqrt{\left(x_{j}-x_{i}\right)^{2}+\left(y_{j}-y_{i}\right)^{2}+\left(z_{j}-z_{i}\right)^{2}}$. 
The force exerted by sensor $s_{j}$ on sensor $s_{i}$ is

- an attractive force if $d_{i j}>D_{t h}$ and is given by:

$$
\overrightarrow{\mathcal{F}}_{i j}=K_{a} \cdot\left(d_{i j}-D_{t h}\right) \cdot \frac{\left(x_{j}-x_{i}, y_{j}-y_{i}, z_{j}-z_{i}\right)}{d_{i j}}
$$

- a replusive force, if $d_{i j}<D_{t h}$ and is equal to

$$
\overrightarrow{\mathcal{F}}_{i j}=K_{r} \cdot\left(D_{t h}-d_{i j}\right) \cdot \frac{\left(x_{i}-x_{j}, y_{i}-y_{j}, z_{i}-z_{j}\right)}{d_{i j}}
$$

- null otherwise, $\left(d_{i j}=D_{t h}\right)$

Hence, the resultant force $\overrightarrow{\mathcal{F}}_{i}$ on $s_{i}$ is computed as the sum of these forces exerted by its 1-hop and 2-hop neighbors:

$$
\overrightarrow{\mathcal{F}}_{i}=\sum_{j} \overrightarrow{\mathcal{F}}_{i j}
$$

Then, node $s_{i}$ moves according to the resultant force to its new position. The new position of sensor $s_{i}$ is given by $\left(x_{i}^{\prime}, y_{i}^{\prime}, z_{i}^{\prime}\right)$ with $x_{i}^{\prime}=x_{i}+F_{i x}, y_{i}^{\prime}=y_{i}+F_{i y}$ and $z_{i}^{\prime}=z_{i}+F_{i z}$.

Notice that the intensity of the resultant force can be large: the node will move a large distance, which can lead to large oscillations that are energy consuming. To overcome this drawback, a sensor node is restricted to move at most a pre-defined distance Distmax at each iteration. To avoid nodes crossing the borders of the 3D area considered, we apply a border force which is a repulsive force. The 3D-DVFA algorithm runs until achieving full area coverage.

\section{PERFormance Evaluation}

In this section, we start by computing the number of nodes needed to cover a $100 m x 100 m x 100 m$ space. Then, we present the simulation parameters adopted to evaluate the performances of 3D-DVFA using two different configurations. Finally, we give the performances evaluation results of 3DDVFA in terms of 3D area coverage and total distance traveled by nodes.

\section{A. Computation of the number of nodes}

The exact number of nodes needed to full cover a 3D space is not known. We propose a lower bound and an upper bound of the number of node needed to cover a cube of length $l=$ $100 \mathrm{~m}$.

$$
\text { Lower bound }=\frac{\text { Cube volume }}{\text { Sphere volume }}=\frac{3 l^{3}}{4 \pi R_{s}^{3}}
$$

Due to borders effect, the lower bound may not be sufficient to fully cover the cube. Then, sensor nodes should not only cover the cube but also its 6 faces and its 12 edges.

$$
\text { Upper bound }=\frac{3 l^{3}}{4 \pi R_{s}^{3}}+6 \frac{l^{2}}{\pi R_{s}^{2}}+12 \frac{l}{R_{s}}
$$

According to Equation 3, our lower bound is equal to 87 nodes and to Equation 4 our upper bound is 271 nodes. In our work, we use 250 nodes to ensure full 3D area coverage and connectivity using the virtual forces strategy.
The regular dedocahedron is on the one hand a regular shape that fits the virtual forces strategy and on the other hand it minimizes the total number of nodes needed to ensure full $3 \mathrm{D}$ area. The number of nodes required by different polyhedrons such that the cube, the hexagonal prism, the rhombic dodecahedron and the truncated octahedron is studied in [9]. The authors define the volumetric quotient to determine the best polyhedron. Let $V$ be the volume of the polyhedron and $R$ is the maximum distance from its center to any vertex, then the volumetric quotient is:

$$
\frac{V}{\frac{4}{3} \pi R^{3}}
$$

The authors proved that the truncated ocatahedron gives the best volumetric quotient with the value of 0.68329 and provides the optimized number of nodes. According to equation 5, the volumetric quotient of the regular dodecahedron is equal to 0.666 which is very close to the value provided by the dodecahedron. Table I, extracted from [9] and completed with the dodecahedron, presents a comparaison between the different polyhedron in terms of volumetric quotient and the number of nodes compared to the truncated octahedron. The regular dodecahedron used in our work requires a number of nodes that exceeds the number required by the truncated octahedron by only $2.59 \%$ which is a neglected value compared with the other polyhedrons.

TABLE I: Volumetric quotient and number of nodes

\begin{tabular}{|c|c|c|}
\hline Polyhedron & Volumetric quotient & $\begin{array}{c}\text { Number of nodes needed } \\
\text { compared to truncated octahedron }\end{array}$ \\
\hline Cube & 0.36755 & $85.9 \%$ more \\
\hline Hexagonal Prism & 0.477 & $43.25 \%$ more \\
\hline Rhombic Dodecahedron & 0.477 & $43.25 \%$ more \\
\hline Regular Dodecahedron & 0.666 & $2.59 \%$ more \\
\hline Truncated Octahedron & 0.68329 & - \\
\hline
\end{tabular}

\section{B. Simulation parameters}

To evaluate performances of our 3D-DVFA algorithm, we implemented it in the Network Simulator NS3 version 3.20. We decided to use NS3 simulator due to its adaptability to different network technologies and to diverse features it offers.

Table II illustrates the simulation parameters used to evaluate the 3D-DVFA algorithm.

\section{3D-DVFA Evaluation}

The goal of 3D-DVFA is to provide a self-deployment that ensures full 3D area coverage and maintains network connectivity. Based on the virtual forces principle and the values of $R_{s}$ and $R_{c}$, if full $3 \mathrm{D}$ area coverage is ensured, network connectivity will be ensured too. Consequently, in this work we evaluate only the coverage ratio with regards to initial configuration and number of nodes deployed.

To determine the coverage ratio, we divide the whole space into $100 m * 100 m * 100 m$ unit cubes. Hence, the coverage ratio is calculated as the ratio of unit cubes whose center is covered by at least one sensor.

As 3D-DVFA algorithm is run by mobile and autonomous 
TABLE II: Simulation parameters

\begin{tabular}{cc}
\hline \hline Toplogy & \\
\hline \hline Sensor nodes & 250 \\
Area size & 100mx 100mx 100m \\
\hline \hline MAC Layer & IEEE $802.11 \mathrm{~b}$ \\
Protocol & $2 \mathrm{Mb} / \mathrm{s}$ \\
Throughput & $26 \mathrm{~m}$ \\
Transmission range $R_{c}$ & $14 \mathrm{~m}$ \\
Sensing range $R_{s}$ & \\
\hline \hline Simulation & $500 \mathrm{~s}$ \\
\hline \hline Result & \\
Simulation time & 0.004 \\
\hline \hline 3D-DVFA & 0.25 \\
Ka & $2.4 \mathrm{~s}$ \\
Kr & $22.27 \mathrm{~m}$ \\
Hello period & 4 \\
Dth & \\
Distmax & average of $20 \mathrm{simulation} \mathrm{runs}$ \\
\hline \hline
\end{tabular}

sensor nodes, the energy consumption due to sensor moves may be high. That is why, we evaluate the total distance traveled by nodes.

We evaluate the coverage rate and the distance traveled using two initial configurations:

- Random configuration: Sensor nodes are scattered randomly in the whole space. See Figure 4a.

- Centered configuration: Sensor nodes are grouped initially around the center of the space, i.e $x \in[25,75], y \in[25,75], z \in[25,75] . \quad$ See Figure $4 \mathrm{~b}$.

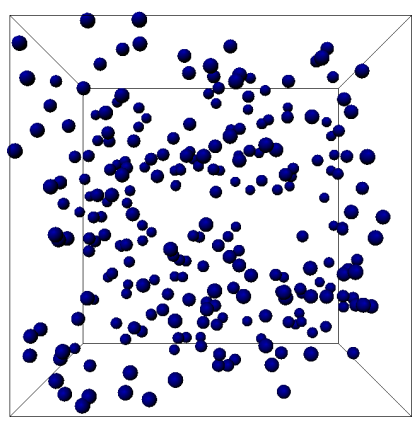

a Initial random configuration.

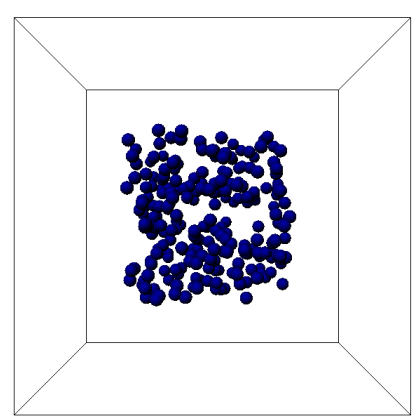

b Initial centered configuration.
Fig. 4: Initial Configurations.

Figure 5 illustrates the final deployment using 250 nodes, obtained with both initial configurations.

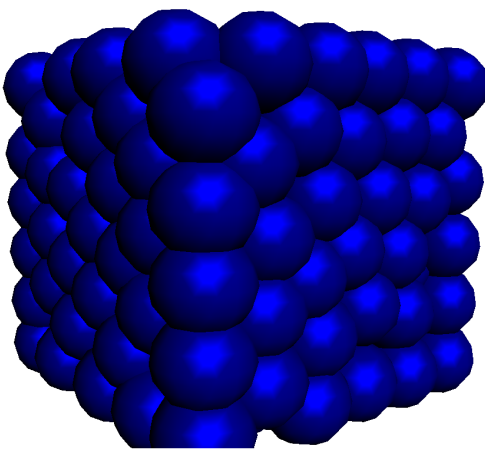

Fig. 5: Final deployment.

1) Evaluation of the coverage rate: Figure 6 illustrates the coverage rate obtained with $3 \mathrm{D}-\mathrm{DVFA}$ as a function of time using 250 nodes in random configuration and centered configuration. In both configurations, sensor deployment based on 3D-DVFA provides full coverage of the 3D area considered (see Figure 5). Figure 6 shows that the coverage rate reaches $99,99 \%$ at time $t=150 \mathrm{~s}$ in both configurations and this rate is still maintained during the remaining simulation time. This can be explained by the spreading of nodes caused by virtual forces. Due to virtual forces, sensor nodes are able to spread in the whole 3D area, reach very quickly full coverage, while maintaining network connectivity.

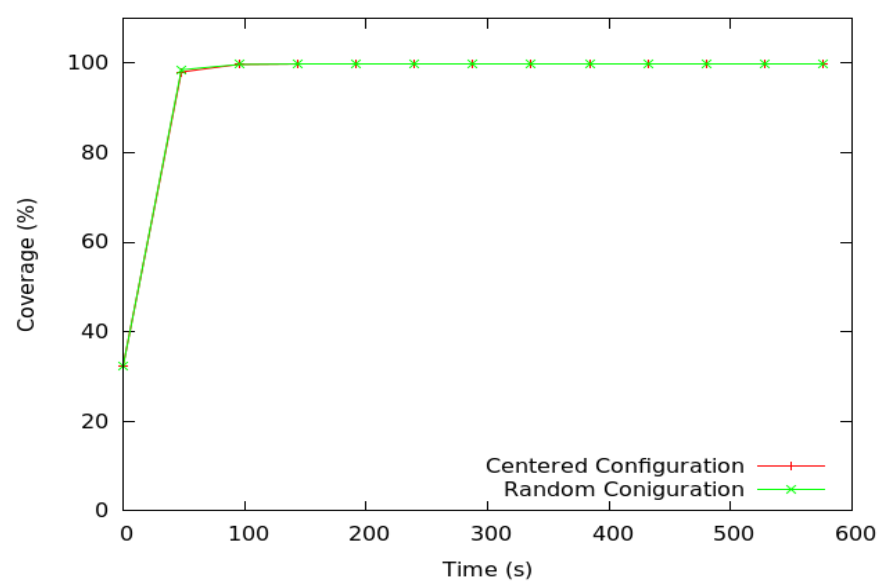

Fig. 6: Coverage rate as function of time

2) Evaluation of the total distance traveled by nodes: Figure 7 illustrates the total distance traveled by nodes as a function of time. The value presented in Figure 7 is the cumulative distance. We can observe that the distance traveled by nodes increases rapidly until $t=100 \mathrm{~s}$ with random configuration and $t=150 \mathrm{~s}$ with centered configuration, after this time the curve of the distance traveled for both configurations increases very slowly. Then, when full coverage has not been reached yet, nodes move in order to reach their final positions. However, when full coverage is ensured, nodes oscillate due to, for instance, border effects and the number of nodes deployed that may be higher than the optimal one. As expected, the distance traveled by nodes in random con- 
figuration is less than the one obtained in the centered configuration. This can be explained by the fact that in the centered configuration sensors are grouped in the center of the 3D area, and in the random configuration they are already spread in the whole 3D area. Thus, sensor nodes move much more with the centered configuration to reach full coverage than with random configuration.

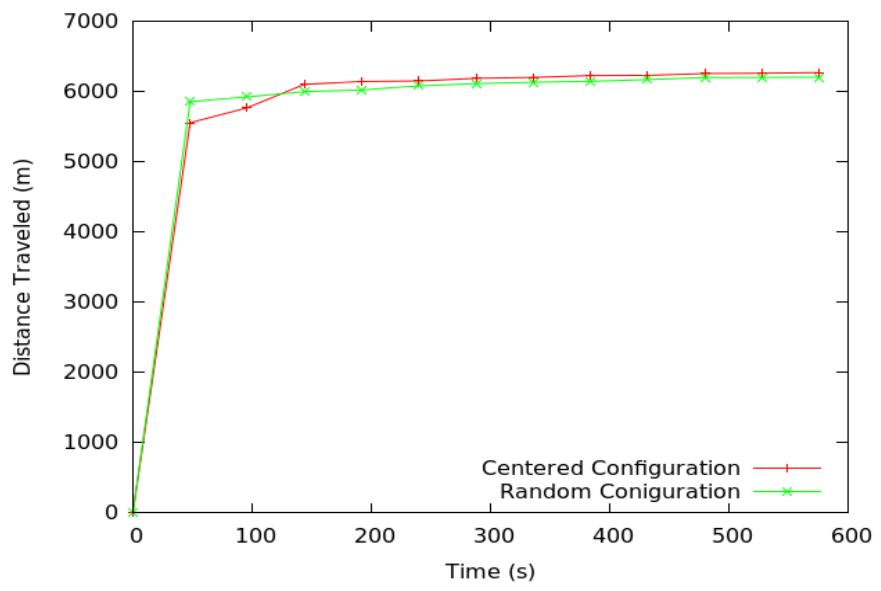

Fig. 7: Total distance traveled by nodes

\section{CONCLUSION AND PERSPECTIVES}

We are interested in mobile wireless sensor networks deployed randomly in a three dimensional space, such as precision agriculture, environmental monitoring, pollutant tracking. The goal consists in redeploying nodes in order to fully cover the whole space while ensuring a connected network. The traditional virtual forces strategy is selected as a reference due to nodes mobility features and its distributed computation. We designed and implemented a 3D algorithm based on virtual forces to ensure full coverage of the space considered and maintain network connectivity. We tuned the value of its parameters to fit $3 \mathrm{D}$ environments.

\section{REFERENCES}

[1] Y. Zou and K. Chakrabarty, "Sensor deployment and target localization based on virtual forces," in INFOCOM 2003. Twenty-Second Annual Joint Conference of the IEEE Computer and Communications. IEEE Societies, vol. 2. IEEE, 2003, pp. 1293-1303.

[2] M. Younis and K. Akkaya, "Strategies and techniques for node placement in wireless sensor networks: A survey," Ad Hoc Networks, vol. 6 , no. 4, pp. 621-655, 2008.

[3] X. Bai, S. Kumar, D. Xuan, Z. Yun, and T. H. Lai, "Deploying wireless sensors to achieve both coverage and connectivity," in Proceedings of the 7th ACM international symposium on Mobile ad hoc networking and computing. ACM, 2006, pp. 131-142.

[4] S. Poduri, S. Pattem, B. Krishnamachari, and G. S. Sukhatme, "Sensor network configuration and the curse of dimensionality," in Proc. Third Workshop on Embedded Networked Sensors (EmNets 2006), Cambridge, MA, USA, 2006.

[5] V. Ravelomanana, "Extremal properties of three-dimensional sensor networks with applications," Mobile Computing, IEEE Transactions on, vol. 3, no. 3, pp. 246-257, 2004.

[6] C.-F. Huang, Y.-C. Tseng, and L.-C. Lo, "The coverage problem in three-dimensional wireless sensor networks," Journal of Interconnection Networks, vol. 8, no. 03, pp. 209-227, 2007.

[7] M. K. Watfa, "Coverage issues in wireless sensor networks," Ph.D. dissertation, University of Oklahoma, 2006.

[8] A. Nauman, "Optimizing coverage in 3d wireless sensor networks," Smart Wireless Sensor Networks, 2010.

[9] S. Alam and Z. J. Haas, "Coverage and connectivity in three-dimensional networks," in Proceedings of the 12th annual international conference on Mobile computing and networking. ACM, 2006, pp. 346-357.

[10] X. Bai, C. Zhang, D. Xuan, and W. Jia, "Full-coverage and kconnectivity $(\mathrm{k}=14,6)$ three dimensional networks," in INFOCOM 2009, IEEE. IEEE, 2009, pp. 388-396.

[11] X. Bai, C. Zhang, D. Xuan, J. Teng, and W. Jia, "Low-connectivity and full-coverage three dimensional wireless sensor networks," in Proceedings of the tenth ACM international symposium on Mobile ad hoc networking and computing. ACM, 2009, pp. 145-154.

[12] X. Li, L. Ci, M. Yang, C. Tian, and X. Li, "Deploying three-dimensional mobile sensor networks based on virtual forces algorithm," in Advances in Wireless Sensor Networks. Springer, 2013, pp. 204-216.

[13] F. Kribi, P. Minet, and A. Laouiti, "Redeploying mobile wireless sensor networks with virtual forces," in Wireless Days (WD), 2009 2nd IFIP. IEEE, 2009, pp. 1-6.

[14] A. Howard, M. J. Matarić, and G. S. Sukhatme, "Mobile sensor network deployment using potential fields: A distributed, scalable solution to the area coverage problem," in Distributed Autonomous Robotic Systems 5. Springer, 2002, pp. 299-308. 